\title{
On a Subposet of the Tamari Lattice
}

\author{
Sebastian A. Csar ${ }^{1}$ \\ Rik Sengupta ${ }^{2}$ \\ ${ }^{1}$ School of Mathematics, University Of Minnesota, Minneapolis, MN, USA \\ ${ }^{2}$ Department of Mathematics, Princeton University, NJ, USA \\ ${ }^{3}$ Department of Mathematics, Massachusetts Institute of Technology, USA
}

Warut Suksompong ${ }^{3}$

\begin{abstract}
We discuss some properties of a subposet of the Tamari lattice introduced by Pallo (1986), which we call the comb poset. We show that three binary functions that are not well-behaved in the Tamari lattice are remarkably well-behaved within an interval of the comb poset: rotation distance, meets and joins, and the common parse words function for a pair of trees. We relate this poset to a partial order on the symmetric group studied by Edelman (1989).

Résumé. Nous discutons d'un subposet du treillis de Tamari introduit par Pallo. Nous appellons ce poset le comb poset. Nous montrons que trois fonctions binaires qui ne se comptent pas bien dans le trellis de Tamari se comptent bien dans un intervalle du comb poset : distance dans le trellis de Tamari, le supremum et l'infimum et les parsewords communs. De plus, nous discutons un rapport entre ce poset et un ordre partiel dans le groupe symétrique étudié par Edelman.
\end{abstract}

Keywords: poset, Tamari lattice, tree rotation

\section{Introduction}

The set $\mathbb{T}_{n}$ of all complete binary trees with $n$ leaves, or, equivalently, parenthesizations of $n$ letters, has been well-studied. Of particular interest here is a partial order on $\mathbb{T}_{n}$, giving the well-studied Tamari lattice, $\mathscr{T}_{n}$. Huang and Tamari (1972) encoded the Tamari order via compenentwise comparison of bracketing vectors. However, while the meet in $\mathscr{T}_{n}$ is characterized by the componentwise minimum of bracketing vectors, the same is not true of the join.

Also of interest is graph underlying the Hasse diagram of $\mathscr{T}_{n}$, which is denoted $\mathscr{R}_{n}$ and called the rotation graph. The rotation graph is the 1-skeleton of the an $(n-2)$-dimensional convex polytope called the associahedron, $K_{n+1}$. The diameter of $\mathscr{R}_{n}$ remains an open question, and understanding meets and joins in the Tamari lattice does not enable one to compute the rotation distance $d_{\mathscr{R}_{n}}\left(T_{1}, T_{2}\right)$ between two trees.

In addition to rotation distance, there is another binary function on $\mathbb{T}_{n}$, which becomes relevant in light of an approach to the Four Color Theorem suggested by Kauffman (1990): : the size of the set ParseWords $\left(T_{1}, T_{2}\right)$ consisting of all words $w \in\{0,1,2\}^{n}$ which are parsed by both $T_{1}$ and $T_{2}$. A word $w$ is parsed by $T$ if the labeling of the leaves of $T$ by $w_{1}, w_{2}, \ldots, w_{n}$ from left to right extends to a proper 3 -coloring with colors $\{0,1,2\}$ of all $2 n-1$ vertices in $T$ such that no two children of the same vertex have the same label and such that no parent and child share the same label. See Figure 11 for an example. 


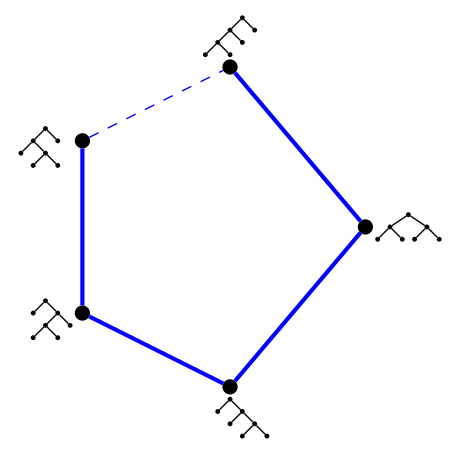

Fig. 1: The comb poset $\mathscr{C}_{4}$

Kauffman (1990) showed that the Four Color Theorem is equivalent to the statement that for all $n$ and all $T_{1}, T_{2} \in \mathbb{T}_{n}$, one has $\left|\operatorname{ParseWords}\left(T_{1}, T_{2}\right)\right| \geq 1$.

Recent work on the ParseWords function by Cooper, Rowland, and Zeilberger (2010) motivated us to investigate a subposet of $\mathscr{T}_{n}$, which we call the (right) comb poset and denote $\mathscr{C}_{n}$. Pallo (2003) first defined $\mathscr{C}_{n}$ and showed that it is a meet-semilattice having the same bottom element as $\mathscr{T}_{n}$. However, meets in the comb poset do not, in general, coincide with meets in the Tamari lattice. The solid edges the diagram in Figure 1 the Hasse diagram of $\mathscr{C}_{4}$. The dashed edge lies in $\mathscr{T}_{4}$ but not in $\mathscr{C}_{4}$.

The intervals of $\mathscr{C}_{n}$ has a number of nice properties which can shed light on the Tamari lattice:

- $\mathscr{C}_{n}$ is ranked and locally distributive, meaning each interval forms a distributive lattice (see Corollary 2.9(i).

- If $T_{1}$ and $T_{2}$ have an upper bound in $\mathscr{C}_{n}$ (or equivalently, if they both lie in some interval), the meet $T_{1} \wedge \mathscr{C}_{n} T_{2}$ and join $T_{1} \vee_{\mathscr{C}_{n}} T_{2}$ are easily described, either in terms of intersection or union of reduced parenthesizations (see Corollary 2.9(i) , or by componentwise minimum or maximum of their bracketing vectors. These operations also coincide with the Tamari meet $\wedge \mathscr{T}_{n}$ and Tamari join $\vee_{\mathscr{T}_{n}}$ (see Theorem 4.5).

- When trees $T_{1}, T_{2}$ have an upper bound in $\mathscr{C}_{n}$, one has (see Theorem 3.1

$$
\begin{aligned}
d_{\mathscr{R}_{n}}\left(T_{1}, T_{2}\right) & =\operatorname{rank}\left(T_{1}\right)+\operatorname{rank}\left(T_{2}\right)-2 \cdot \operatorname{rank}\left(T_{1} \wedge_{\mathscr{C}_{n}} T_{2}\right) \\
& =2 \cdot \operatorname{rank}\left(T_{1} \vee_{\mathscr{C}_{n}} T_{2}\right)-\left(\operatorname{rank}\left(T_{1}\right)+\operatorname{rank}\left(T_{2}\right)\right) \\
& =\operatorname{rank}\left(T_{1} \vee_{\mathscr{C}_{n}} T_{2}\right)-\operatorname{rank}\left(T_{1} \wedge_{\mathscr{C}_{n}} T_{2}\right),
\end{aligned}
$$

where, for any $T \in \mathbb{T}_{n}, \operatorname{rank}(T)$ refers to the rank of $T$ in $\mathscr{C}_{n}$.

- Furthermore, for $T_{1}, T_{2}$ having an upper bound in $\mathscr{C}_{n}$, one has (see Theorem 6.6)

$$
\operatorname{ParseWords}\left(T_{1}, T_{2}\right)=\operatorname{ParseWords}\left(T_{1} \wedge_{\mathscr{C}_{n}} T_{2}, T_{1} \vee_{\mathscr{C}_{n}} T_{2}\right),
$$

with cardinality $3 \cdot 2^{n-1-k}$, where $k=\operatorname{rank}\left(T_{1} \vee_{\mathscr{C}_{n}} T_{2}\right)-\operatorname{rank}\left(T_{1} \wedge_{\mathscr{C}_{n}} T_{2}\right)$. 
There are a number of well-known related order-preserving surjections from the (right) weak order on the symmetric group $\mathfrak{S}_{n}$ to $\mathscr{T}_{n+1}$. (See, for instance, Loday and Ronco (2002) and Hivert et al. (2005) for discussion of such maps.) Section 5 discusses how one of these maps restricts to an orderpreserving surjection from $\mathscr{E}_{n}$ to $\mathscr{C}_{n+1}$, where $\mathscr{E}_{n}$ is a subposet of the weak order defined by Edelman (1989). Additionally, this surjection is a distributive lattice morphism on each interval of $\mathscr{C}_{n+1}$ (see Theorem 5.12.

Except when there is risk for confusion, the subscripts will be dropped from $\wedge, \vee,>$ and $<$ when denoting meet, join, greater than and less than, respectively, in $\mathscr{C}_{n}$. Additionally, $\operatorname{rank}(T)$ will denote the rank of $T$ in $\mathscr{C}_{n}$. Much of the notation in Section 6 is from Cooper et al. (2010). This extended abstract is an announcement of the results of Csar et al. (2010), which is more detailed and contains proofs of the results given here.

\section{The Comb Poset and Distributivity}

Pallo (2003) defined the comb poset defined the comb poset via covering relations consisting of a left tree rotation centered on the right arm of the tree. This section presents a definition of the comb poset in terms of reduced parenthesizations and discusses some properties of the poset and its intervals.

Definition 2.1 For each binary tree $T \in \mathbb{T}_{n}$, consider the usual parenthesization of its leaves $a_{1}, a_{2}, \ldots, a_{n}$. Then, delete all pairs of parentheses that enclose the leaf $a_{n}$. Call the resulting parenthesization the reduced parenthesization of $T$, denoted $R P_{T}$. Define an element of $R P_{T}$ to be either an unparenthesized leaf in $R P_{T}$, or any pair of parentheses $J$ in $R P_{T}$ (together with all enclosed leaves and internal parenthesizations) which is not enclosed by some other pair of parentheses in $R P_{T}$.

Example 2.2 The reduced parenthesization of the tree in Figure 2 is $a_{1}\left(\left(a_{2} a_{3}\right) a_{4}\right)\left(a_{5} a_{6}\right) a_{7}$. The reduced parenthesization of this tree has four elements, given by $a_{1},\left(\left(a_{2} a_{3}\right) a_{4}\right),\left(a_{5} a_{6}\right)$, and $a_{7}$. Note that $a_{n}$ by itself is always an element of the reduced parenthesization of any tree in $\mathbb{T}_{n}$.

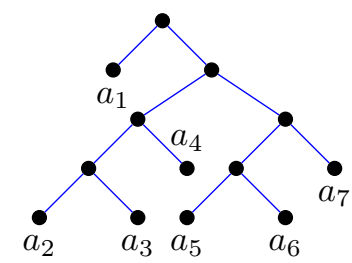

Fig. 2: The tree $a_{1}\left(\left(a_{2} a_{3}\right) a_{4}\right)\left(a_{5} a_{6}\right) a_{7}$.

Definition 2.3 Define the (right) comb poset of order $n$ to be the poset whose elements are given by elements from $\mathbb{T}_{n}$, with $T_{1} \leq T_{2}$ iff each pair of parentheses in $R P_{T_{1}}$ appears in $R P_{T_{2}}$. Denote the comb poset by $\mathscr{C}_{n}$.

One could reverse the inclusion of the parentheses pairs in the above definition and obtain a left comb poset, for which analogous results hold.

From the definition, one sees that $\mathscr{C}_{n}$ has a unique minimal element-the tree whose reduced parenthesization contains no parentheses. This tree is called the right comb tree, denoted $\mathrm{RCT}(() n)$. 
Example 2.4 The Hasse diagram of the right comb poset of order 5 is shown in Figure 3 For the sake of a cleaner diagram, the leaf $a_{i}$ is labeled by $i$ in Figure 3 for $i \in\{1,2,3,4,5\}$.

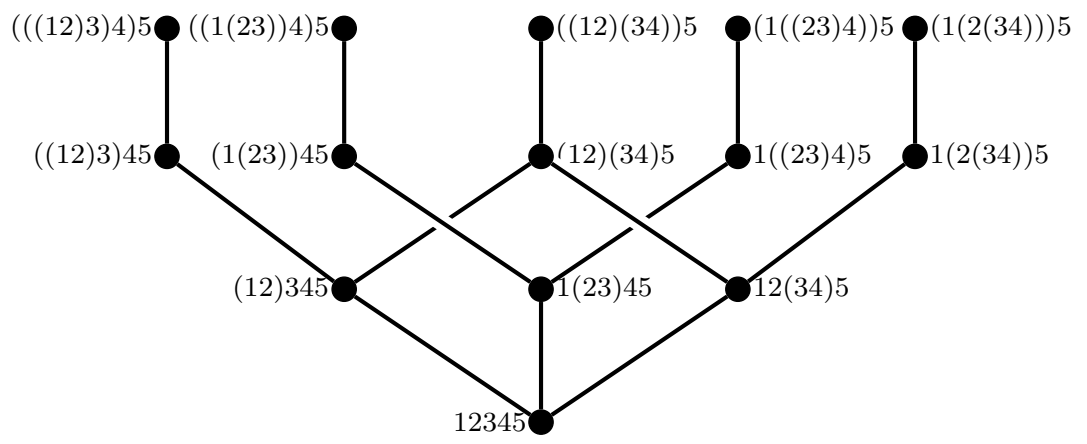

Fig. 3: The Hasse diagram of $\mathscr{C}_{5}$

Example 2.5 $\mathrm{RCT}(5)$, the right comb tree of order 5 , is shown in Figure 4 The nodes labeled $a_{1}, \ldots, a_{5}$ are the leaves of the tree, and $b_{6}, \ldots, b_{9}$ are the internal vertices. Note that the structure of the left comb tree of order 5 is given by the reflection of the right comb tree about the vertical axis.

To consider the intervals of $\mathscr{C}_{n}$, one defines another poset, the reduced pruned poset. Recall that the right arm of a tree, $T$, is the connected acyclic group induced by the vertices of $T$ that lie in the left subtree of no other vertex of $T$.

There is a well known operation on complete binary trees, called pruning, where one removes all leaves from an $n$-leaf binary tree, obtaining an "incomplete" tree on $n-1$ vertices. Pruning gives a bijection between $n$-leaf trees and (possibly incomplete) binary trees with $n-1$ vertices.

Definition 2.6 For a tree $T \in \mathbb{T}_{n}$, the reduced pruned poset of $T$, denoted $P_{T}$, is the poset of pairs of parentheses in $R P_{T}$ ordered by inclusion. Its Hasse diagram is obtained by pruning $T$, removing the right arm and removing edges incident to the right arm.

Example 2.7 Consider the tree of Figure 2 given by the reduced parenthesization $a_{1}\left(\left(a_{2} a_{3}\right) a_{4}\right)\left(a_{5} a_{6}\right) a_{7}$. Figure 5 depicts its "pruned" form and the corresponding reduced pruned poset $P_{T}$.

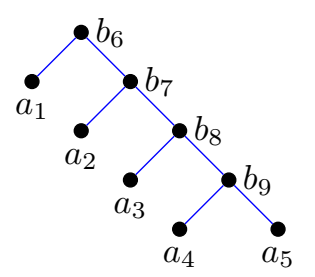

Fig. 4: RightCombTree(5) 


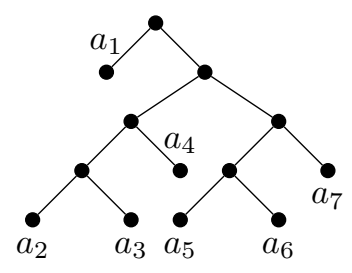

(a) $T$

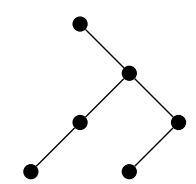

(b) $T$ pruned

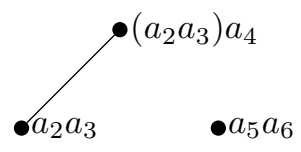

(c) $P_{T}$

Fig. 5: The tree $a_{1}\left(\left(a_{2} a_{3}\right) a_{4}\right)\left(a_{5} a_{6}\right) a_{7}$, its pruned tree and reduced pruned poset.

The following proposition gives a key relationship between $P_{T}$ and $\mathscr{C}_{n}$.

Proposition 2.8 For any $T \in \mathbb{T}_{n}$, the interval $[\operatorname{RCT}(n), T]_{\mathscr{C}_{n}}$ is isomorphic to the lattice of order ideals in the reduced pruned poset of $T$, ordered by inclusion. In other words, for any tree $T$,

$$
[\operatorname{RCT}(n), T]_{\mathscr{C}_{n}} \cong J\left(P_{T}\right)
$$

That the principal order ideal in $\mathscr{C}_{n}$ of any tree $T$ is a distributive lattice yields a number of immediate corollaries.

\section{Corollary 2.9}

(i) Any interval in $\mathscr{C}_{n}$ is a distributive lattice, with the reduced parenthesizations of the join and meet of trees $T_{1}$ and $T_{2}$ in an interval given by the ordinary union and intersection of parenthesis pairs from $R P_{T_{1}}$ and $R P_{T_{2}}$.

(ii) In $\mathscr{C}_{n}, T_{1}$ covers $T_{2}$ iff $R P_{T_{1}}$ can be obtained from $R P_{T_{2}}$ by adding one parenthesis pair.

(iii) $\mathscr{C}_{n}$ is a ranked poset, with the rank of any tree $T$ in $\mathscr{C}_{n}$ given by the number of parenthesis pairs in $R P_{T}$.

(iv) For any two trees $T_{1}$ and $T_{2}$ that are in the same interval of $\mathscr{C}_{n}$, we have

$$
\operatorname{rank}\left(T_{1}\right)+\operatorname{rank}\left(T_{2}\right)=\operatorname{rank}\left(T_{1} \wedge T_{2}\right)+\operatorname{rank}\left(T_{1} \vee T_{2}\right)
$$

(v) For any tree $T \in \mathbb{T}_{n}$ of rank $k$, the length of the right arm of $T$ is $n-1-k$.

The covering relation described in Corollary 2.9(ii) corresponds precisely to the so-called right arm rotations used by Pallo (2003) to define the comb poset.

\section{Distances in $\mathscr{C}_{n}$ and $\mathscr{R}_{n}$}

The diameter of the Tamari lattice (or, more precisely, the rotation graph $\mathscr{R}_{n}$ ) is an open question. Sleator et al. (1988) show that the diameter is at most $2 n-6$. When one restricts one's attention to trees lying in the same interval of the comb poset, one can obtain precise information about distances not only in $\mathscr{C}_{n}$, but $\mathscr{R}_{n}$ as well. 
Theorem 3.1 If $T_{1}$ and $T_{2}$ are two trees in some interval in $\mathscr{C}_{n}$, then the shortest distance between them along the edges of the rotation graph $\mathscr{R}_{n}$ is given by

$$
\begin{aligned}
d_{\mathscr{R}_{n}}\left(T_{1}, T_{2}\right) & =\operatorname{rank}\left(T_{1}\right)+\operatorname{rank}\left(T_{2}\right)-2 \cdot \operatorname{rank}\left(T_{1} \wedge T_{2}\right) \\
& =d_{\mathscr{R}_{n}}\left(T_{1}, T_{2}\right)=2 \cdot \operatorname{rank}\left(T_{1} \vee T_{2}\right)-\operatorname{rank}\left(T_{1}\right)-\operatorname{rank}\left(T_{2}\right),
\end{aligned}
$$

where the ranks are those in the comb poset. Moreover, such a shortest path uses only edges in $\mathscr{C}_{n}$.

It can easily be shown from the above that the diameter of $\mathscr{R}_{n}$ is at most $2 n-4$, not quite as tight as the bound of Sleator et al. (1988).

\section{Tamari Meets and Joins for two Trees in Some Interval}

Corollary 2.9(i) characterizes the meets and (when they exist) joins in the comb poset. It is natural to ask how the meets and joins in the comb poset relate to the meets and joins of the Tamari lattice. We will refer to meets and joins in $\mathscr{T}_{n}$ as the "Tamari meet" and "Tamari join" to avoid confusion.

While $\mathscr{C}_{n}$ is a meet semilattice, so any two trees do have a meet in $\mathscr{C}_{n}$, their meet does not necessarily coincide with their Tamari meet. For example, consider $T_{1}=\left(\left(\left(a_{1} a_{2}\right) a_{3}\right) a_{4}\right) a_{5}$ and $T_{2}=$ $\left(\left(a_{1}\left(a_{2} a_{3}\right)\right) a_{4}\right) a_{5}$. Their Tamari meet is $T_{2}$, while their meet in $\mathscr{C}_{n}$ is the right comb tree.

However, if we, once again, restrict our attention to trees lying in some interval of the comb poset, i.e. pairs of trees that have a join in $\mathscr{C}_{n}$, the meet and join in the comb poset $d o$ coincide with the Tamari meet and join.

To see this relationship, one characterizes the meet and join not in terms of parenthesis pairs as before, but using bracketing vectors, as introduced by Huang and Tamari (1972).

Recall that one can prune an $n$-leaf binary tree to obtain a (possibly incomplete) binary tree on $n-1$ vertices. Furthermore, there is a well-known natural numbering of the vertices of the pruned tree using $1,2, \ldots, n-1$, in which a vertex receives a higher number than any vertex in its left subtree, but a lower one than any vertex in its right subtree. This labeling is unique. It is called the in-order labeling of a pruned tree on $n-1$ vertices.

Example 4.1 Figure 6 shows a pruned tree on 8 vertices, corresponding to the 9-leaf binary tree whose reduced parenthesization is $\left(\left(a_{1} a_{2}\right)\left(a_{3} a_{4}\right)\right) a_{5}\left(a_{6}\left(a_{7} a_{8}\right)\right) a_{9}$, is labeled in the in-order labeling.

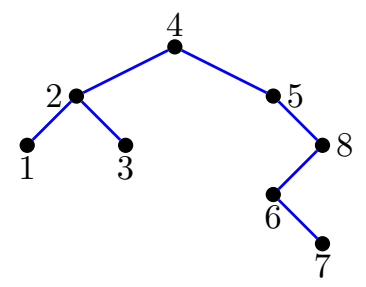

Fig. 6: The pruned tree for $\left(\left(a_{1} a_{2}\right)\left(a_{3} a_{4}\right)\right) a_{5}\left(a_{6}\left(a_{7} a_{8}\right)\right) a_{9}$ labeled with the in-order labeling.

Definition 4.2 Consider the "pruned" binary tree representation of some tree $T \in \mathbb{T}_{n}$, and number the $n-1$ vertices by the in-order labeling. Then, the bracketing vector for $T,\langle T\rangle=\left\langle b_{1}(T), \ldots, b_{n-1}(T)\right\rangle$, 
has $b_{j}(T)$ equal to the number of vertices in the left subtree of the vertex labeled $j$ in the pruned tree. In particular, the first coordinate of a bracketing vector is always 0.

Example 4.3 The bracketing vector for the tree in Figure 6 is the 8-tuple $(0,1,0,3,0,0,0,2)$.

Theorem 4.4 (Pallo (1986, Theorem 2)) For two $n$-leaf binary trees $T$ and $T^{\prime}$, one has $T \leq T^{\prime}$ if and only if the bracketing vector of $T$ is component-wise less than or equal to the bracketing vector of $T^{\prime}$. Furthermore, the bracketing vector for the meet of two trees in the Tamari lattice corresponds to the componentwise minimum of the bracketing vectors of the two trees.

It is not the case in general that the componentwise maximum of bracketing vectors corresponds to the Tamari join. However, when two trees lie in an interval of the comb poset, this property holds.

Theorem 4.5 Let $\langle T\rangle$ denote the bracketing vector for $T$. Let $T_{1}$ and $T_{2}$ be arbitrary trees in the same interval of $\mathscr{C}_{n}$. Then, their meet and join in $\mathscr{C}_{n}$ are given by the trees corresponding respectively to the componentwise minimum and the componentwise maximum of $\left\langle T_{1}\right\rangle$ and $\left\langle T_{2}\right\rangle$. Moreover, their meet and join in the comb post coincide with their Tamari meet and join.

\section{Relation with a Poset of Edelman}

Connections between the Tamari lattice and weak order on $\mathfrak{S}_{n}$ have been the subject of much previous study. In particular, it is well-known (see, for instance, Hivert et al. (2005) ) that the subposet of the weak order induced by the 231-avoiding permutations is isomorphic to the Tamari lattice. It should be noted that Hivert et al. (2005) and Loday and Ronco (2002) consider the dual lattice to $\mathscr{T}_{n}$.

Edelman (1989) introduced a subposet of the right weak order on the symmetric group $\mathfrak{S}_{n}$. Although this poset is not a lattice, the intervals are each known to be distributive lattices, as is the case for the comb poset.

Definition 5.1 The right weak order on $\mathfrak{S}_{n}$ is a partial ordering of the elements of $\mathfrak{S}_{n}$ defined as the transitive closure of the following covering relation: a permutation $\sigma$ covers a permutation $\tau$ if $\sigma$ is obtained from $\tau$ by a transposition of two adjacent elements of the one line notation of $\tau$ introducing an inversion.

Edelman imposed an additional constraint on this ordering, under which $\sigma$ covers $\tau$, if, after the transposition of $x_{j}$ and $x_{j+1}$ as above, nothing to the left of $x_{j+1}$ in $\sigma$ is greater than $x_{j+1}$. This restriction results in a subposet of the right weak ordering on $\mathfrak{S}_{n}$. Denote this poset by $\mathscr{E}_{n}$.

Example 5.2 Figure 7 depicts the Hasse diagram of $\mathscr{E}_{3}$, with an additional dashed edge indicating the extra order relation in the right weak order on $\mathfrak{S}_{3}$.

There are several closely-related maps connecting the weak order to the Tamari lattice, sending permutations to pruned binary trees. Here, we will use the map inserting a permutation into a binary search tree. (This is nearly the map of Hivert et al. (2005), but they first reverse the permutation since they care considering the dual to our Tamari lattice.) 


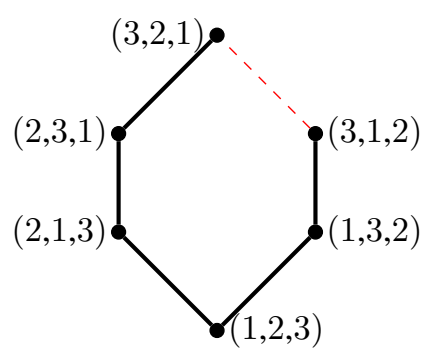

Fig. 7: Edelman's Poset $\mathscr{E}_{3}$.

Definition 5.3 Define a map $p: \mathfrak{S}_{n} \rightarrow\{$ pruned trees on $n$ vertices $\}$ recursively as follows. For $x \in \mathfrak{S}_{1}$, $p(x)$ is the tree with a single vertex. Then, for $n>1$ and $x \in \mathfrak{S}_{n}$, define

$$
p(x)=
$$

where $x_{<}=\left(x_{i_{1}}, \ldots, x_{i_{k}}\right)$ where $i_{1}<\cdots<i_{k}$ are the indices of all elements of $x$ less than $x_{1}$ and $x_{>}$ is defined similarly for elements of $x$ greater than $x_{1}$. Extend $p$ to a map $\mathfrak{S}_{n} \rightarrow \mathbb{T}_{n+1}$, also called $p$, by attaching leaves to $p(x)$ to give a binary tree (in other words, "unpruning" $p(x)$ ).

Remark 5.4 Amending the definition of $p$ slightly so that the root of $p(x)$ is labeled by $x_{1}$ results in the pruned tree having the in-order labeling (see Section 4), i.e. the result of inserting $x$ into a binary search tree. (See Knuth(1973).)

Example 5.5 Figure 8 shows $p: \mathfrak{S}_{4} \rightarrow$ \{pruned trees with 4 vertices $\}$. Permutations having the same image are circled.

Theorem 5.6 The map $p: \mathfrak{S}_{n} \rightarrow \mathbb{T}_{n+1}$ gives an order-preserving surjection from $\mathscr{E}_{n}$ to $\mathscr{C}_{n+1}$.

The map $p$ is a lattice morphism between principal ideals in $\mathscr{E}_{n}$ and principal ideals in $\mathscr{C}_{n+1}$, which can be understood as the Birkhoff-Priestly dual to two posets associated with a permutation. Edelman (1989) defined the following order on the inversion set of a permutation $\sigma$.

Definition 5.7 For a permutation $\sigma$, define $I(\sigma):=\left\{(j, i): j>i\right.$ and $\left.\sigma^{-1}(j)<\sigma^{-1}(i)\right\}$. Order $I(\sigma)$, with $(k, \ell) \geq(j, i)$ if and only if $k \geq j$ and $\sigma^{-1}(\ell) \leq \sigma^{-1}(i)$. In a slight abuse of notation, the poset $(I(\sigma),<)$ shall be referred to as $I(\sigma)$ as well.

Theorem 5.8 (Edelman (1989, Theorem 2.13)) $[e, w]_{\mathscr{E}_{n}} \simeq J(I(w))$, where $[e, w]_{\mathscr{E}_{n}}=\left\{v \in \mathfrak{S}_{n}\right.$ : $\left.v \leq_{\mathscr{E}_{n}} w\right\}$, via $v \mapsto I(v)$.

Definition 5.9 Fix a permutation $w \in \mathfrak{S}_{n}$. Let $T_{w}$ be the image of $w$ under the pruned tree map, $p$. Recall the reduced pruned poset from Definition 2.6. Here it will be useful to label its vertices by the labels they have in $T_{w}$, rather than by parentheses. Define a map $f: P_{T_{w}} \rightarrow I(w)$ as follows: $f(j)=(i, j)$, where $i$ is the smallest label of a vertex of $T_{w}$ such that $j$ lies in the left subtree of $i$. 

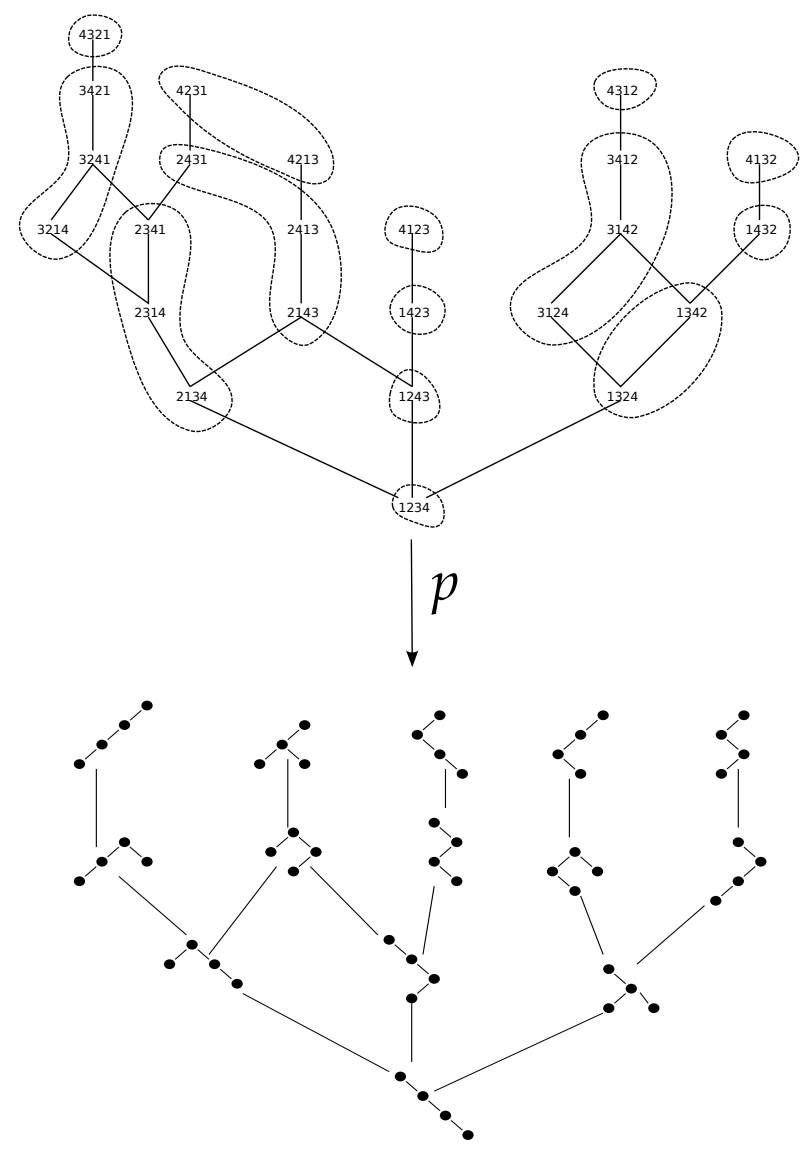

Fig. 8: The map $p: \mathfrak{S}_{4} \rightarrow$ \{pruned trees with 4 vertices $\}$.

Example 5.10 Suppose $w=(4,9,2,1,8,3,6,7,5) \in \mathfrak{S}_{9}$. Figure 9 depicts $T_{w}, P_{T_{w}}$ and $I(w)$, with the image of $f$ indicated in $I(w)$.

One can now understand how $p$ relates intervals in $\mathscr{E}_{n}$ to intervals in $\mathscr{C}_{n+1}$.

Definition 5.11 Let $P_{1}, P_{2}$ be two posets and suppose $\phi: P_{1} \rightarrow P_{2}$ is order-preserving. Then $\phi$ induces a map $J(\phi): J\left(P_{2}\right) \rightarrow J\left(P_{1}\right)$ defined by $J(\phi)(I)=\phi^{-1}(I)$. One calls $J(\phi)$ the Birkhoff-Priestley dual to $f$. In fact, $J(\phi)$ is a lattice morphism.

Theorem 5.12 For each $w \in \mathfrak{S}_{n}$, the map $f: P_{T_{w}} \rightarrow I(w)$ defined in Definition 5.9 is order-preserving and Birkhoff-Priestley dual to the map $p:[e, w]_{\mathscr{E}_{n}} \rightarrow J\left(P_{T_{w}}\right)$. In particular, $p: \mathscr{E}_{n} \rightarrow \mathscr{C}_{n+1}$ becomes a lattice morphism when restricted to any interval in $\mathscr{E}_{n}$.

Example 5.13 Figure 10 depicts Theorem 5.12 on the interval $[e, 4213]_{\mathscr{E}_{n}}$. 

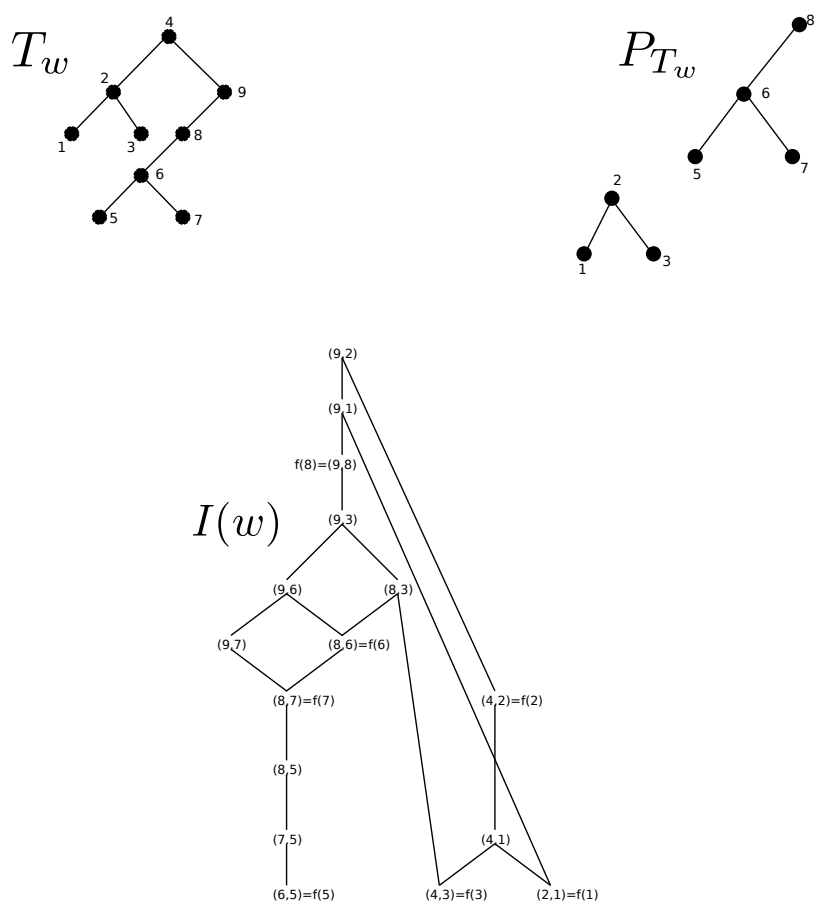

Fig. 9: $T_{w}, P_{T_{w}}$ and $I(w)$ with the image of $f$ indicated.

\section{The ParseWords Function for the Comb Poset}

Recall that $w \in \operatorname{ParseWords}(T)$ means that $T$ admits a labeling of its vertices by $0,1,2$ such that the leaves are labeled by the word $w$, the children of each vertex have distinct labels and no vertex has the same label as either of its children. Kauffman (1990) showed that the Four Color Theorem is equivalent to ParseWords $\left(T_{1}, T_{2}\right) \neq \emptyset$ for all $T_{1}, T_{2} \in \mathbb{T}_{n}$ for any $n \in \mathbb{N}$. It was further recent work by Cooper et al. (2010) seeking a "small" proof of the Four Color Theorem that led us to first consider the comb poset. The number of parsewords for any two trees having a common upper bound in $\mathscr{C}_{n}$ can be computer precisely.

Example 6.1 An example of two trees both parsing 010 is shown in Figure 11.

Example 6.2 The common parsewords for the trees in Figure 11 are 101, 202, 010, 212, 020, 121.

Proposition 6.3 For $T \in \mathbb{T}_{n}$, one has $|\operatorname{ParseWords}(T)|=3 \cdot 2^{n-1}$.

To simplify notation, let $T_{\leq b}$ be the subtree of a tree $T$ having the vertex $b$ as its root.

Proposition 6.4 (Common root property, Cooper et al. (2010, Proposition 2)) If two trees $T_{1}, T_{2} \in \mathbb{T}_{n}$ parse the same word, then their roots receive the same label when the trees are labeled with a common parseword. Hence, iffor $T_{1}, T_{2} \in \mathbb{T}_{n}$, there are vertices $b_{i}$ in $T_{1}$ and $b_{j}$ in $T_{2}$ such that $T_{1_{\leq b_{i}}}$ and $T_{2_{\leq b_{j}}}$ 

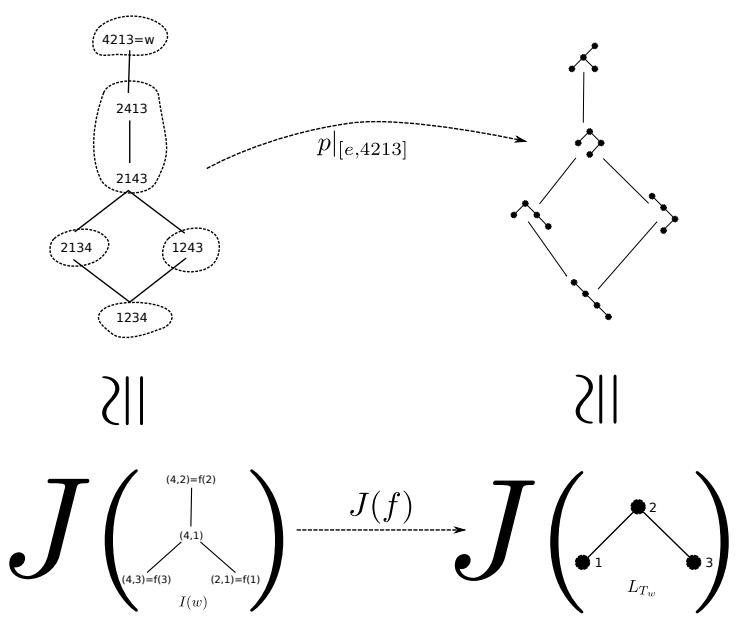

Fig. 10: $\left.p\right|_{[e, 4213]}$ and $J(f)$ for the interval $[e, 4213]_{\mathscr{E}_{n}}$.

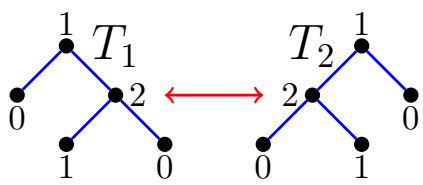

Fig. 11: Both trees parse 010.

have precisely the same leaves (i.e. both the dangling subtrees contain precisely the leaves $m_{1}$ through $m_{2}$, for some natural numbers $m_{1}<m_{2} \leq n$ ), then $b_{i}$ and $b_{j}$ receive the same label if we label the trees with a common parse word.

In particular, for any two trees that differ by a rotation (be it on the right arm or not) there is a set of leaves such that both trees have a subtree with precisely these leaves. With this in mind, one can compute the number of common parsewords for two trees lying in an interval of $\mathscr{C}_{n}$.

Theorem 6.5 Suppose $T_{1}<T<T_{2}$ in $\mathscr{C}_{n}$. Then ParseWords $\left(T_{1}, T_{2}\right)=\operatorname{ParseWords}\left(T_{1}, T_{2}, T\right)$.

Theorem 6.6 Suppose $T_{1}$ and $T_{2}$ have an upper bound in $\mathscr{C}_{n}$. Then

$$
\operatorname{ParseWords}\left(T_{1}, T_{2}\right)=\operatorname{ParseWords}\left(T_{1} \wedge T_{2}, T_{1} \vee T_{2}\right) .
$$

Furthermore, if $\operatorname{rank}\left(T_{1} \vee T_{2}\right)-\operatorname{rank}\left(T_{1} \wedge T_{2}\right)=k$, then

$$
\left|\operatorname{ParseWords}\left(T_{1}, T_{2}\right)\right|=\left|\operatorname{ParseWords}\left(T_{1} \wedge T_{2}, T_{1} \vee T_{2}\right)\right|=3 \cdot 2^{n-1-k} .
$$

In particular, since $\mathscr{C}_{n}$ has $n$ ranks, any two trees with an upper bound in $\mathscr{C}_{n}$ have a common parseword. 


\section{Acknowledgements}

This research was carried out at the School of Mathematics, University of Minnesota - Twin Cities, under the supervision of Vic Reiner and Dennis Stanton with funding from NSF grant DMS-1001933. We would like to thank Vic Reiner and Dennis Stanton for their invaluable guidance and support, Bobbe Cooper for giving a talk introducing us to this problem, Nathan Williams for his words of advice and continual assessment of our progress, and Alan Guo for his input regarding the sizes of ranks in $\mathscr{C}_{n}$.

\section{References}

B. Cooper, E. Rowland, and D. Zeilberger. Toward a language theoretic proof of the Four Color Theorem, 2010. arXiv:1006.1324v1 [math.CO].

S. A. Csar, R. Sengupta, and W. Suksompong. On a subposet of the tamari lattice, 2010. arXiv:1108.5690v1 [math.CO].

P. H. Edelman. Tableaux and chains in a new partial order of $S_{n}$. J. Combin. Theory Ser. A, 51(2): 181-204, 1989.

F. Hivert, J.-C. Novelli, and J.-Y. Thibon. The algebra of binary search trees. Theoret. Comput. Sci., 339 (1):129-165, 2005.

S. Huang and D. Tamari. Problems of associativity: A simple proof for the lattice property of systems ordered by a semi-associative law. J. Combinatorial Theory Ser. A, 13:7-13, 1972.

L. H. Kauffman. Map coloring and the vector cross product. J. Combin. Theory Ser. B, 48(2):145-154, 1990.

D. E. Knuth. The Art of Computer Programming, volume 3: Sorting and Searching. Addison-Wesley Publishing Company, Reading, Massachusetts, 1973.

J.-L. Loday and M. O. Ronco. Order structure on the algebra of permutations and of planar binary trees. J. Algebraic Combin., 15(3):253-270, 2002.

J. M. Pallo. Enumerating, ranking and unranking binary trees. Comput. J., 29(2):171-175, 1986.

J. M. Pallo. Right-arm rotation distance between binary trees. Inform. Process. Lett., 87(4):173-177, 2003. doi: 10.1016/S0020-0190(03)00283-7.

D. D. Sleator, R. E. Tarjan, and W. P. Thurston. Rotation distance, triangulations, and hyperbolic geometry. J. Amer. Math. Soc., 1(3):647-681, 1988. doi: 10.2307/1990951. 